Georgia State University

ScholarWorks @ Georgia State University

\title{
Trading Behavior Between Conspecifics in Chimpanzees, Pan Troglodytes
}

Sarah F. Brosnan

Georgia State University, sbrosnan@gsu.edu

Michael J. Beran

Georgia State University, mberan1@gsu.edu

Follow this and additional works at: https://scholarworks.gsu.edu/psych_facpub

Part of the Psychology Commons

\section{Recommended Citation}

Brosnan, S.F. \& Beran, M.J. (2009). Trading behavior between conspecifics in chimpanzees, Pan troglodytes. Journal of Comparative Psychology, 123(2), 181-194. doi: 10.1037/a0015092

This Article is brought to you for free and open access by the Department of Psychology at ScholarWorks @ Georgia State University. It has been accepted for inclusion in Psychology Faculty Publications by an authorized administrator of ScholarWorks @ Georgia State University. For more information, please contact scholarworks@gsu.edu. 


\title{
Running head: TRADE BETWEEN CONSPECIFICS
}

Trading behavior between conspecifics in chimpanzees, Pan troglodytes

\author{
Sarah F. Brosnan \\ Georgia State University \\ Michael J. Beran \\ Georgia State University
}




\begin{abstract}
Bartering of commodities between individuals is a hallmark of human behavior that is not commonly seen in other species. This is difficult to explain because barter is mutually beneficial, and appears to be within the cognitive capabilities of many species. It may be that other species do not recognize the gains of trade, or that they do not experience conditions (e.g., low risk) in which barter is most beneficial. To answer these questions, we instituted a systematic study of chimpanzees' ability to barter with each other when doing so materially benefits them. Using tokens derived from symbols they have used since infancy, pairs of adult chimpanzees could trade between themselves to obtain tokens needed to get foods. Subjects flexibly used the tokens to obtain foods from an experimenter; however, they did not spontaneously trade with their partner. After extensive training, subjects engaged in accurate trade behavior as long as an experimenter enforced the structure of the interaction; however, trade between partners disappeared when this enforcement was removed. We discuss possible reasons for these findings as well as implications for the evolution of barter across the primate lineage.
\end{abstract}


Trade between conspecifics in chimpanzees, Pan troglodytes

"It [barter, to exchange one thing for another] is common to all men, and to be found in no other race of animals, which seem to know neither this nor any other species of contracts... Nobody ever saw a dog make a fair and deliberate exchange of one bone for another with another dog.”

Adam Smith, The Wealth of Nations

One feature that marks all human societies apart from those of other species is the presence of extensive exchange, trade, and barter (Chapman, 1980; Ofek, 2001). All human societies studied thus far have some form of barter, even between groups which do not otherwise get along (Chapman, 1980) and in the most difficult of circumstances, such as concentration camps and prisons (see overview in Ofek, 2001). In practice, barter can be engaged in for both economic and ceremonial purposes, although it is the former on which we focus in this article. In experimental studies of barter between anonymous individuals, two features have emerged as critical. First, individuals must trust each other (a feature which is more prevalent in non-anonymous situations; Berg, Dickhaut, \& McCabe, 1995), and second, individuals must communicate their intention to barter (Crockett, Smith \& Wilson, in press).

In principle, these two features should not exclude other species from the ability to barter. Although other species lack language, they do communicate and often engage in behavior that appears related to trade, such as food sharing and the exchange of services (e.g. Mitani, 2006; de Waal, 1989). However, barter, in the human sense of actively trading one item for another, has not been documented in animals living in wild 
groups and only in sporadic instances in captivity (Paquette, 1992). Thus, an obvious question is whether barter is a uniquely human activity, or one for which we can find some roots in other species. Understanding this may help us to better understand the environmental context that allowed barter to emerge and thrive to the extent we see in human societies. To begin to address these questions, we investigated the trading abilities of one of our closest living relatives, the chimpanzee (Pan troglodytes), in a controlled experimental setting.

Chimpanzees are good candidates to possess the ability to barter, which we here operationally defined as the voluntary giving of an item in one's possession to another individual, while receiving within a short time span (on the order of seconds or minutes) a second item from the other individual, presumably in return. Aside from being our closest evolutionary cousin, chimpanzees exhibit advanced cognitive capabilities (e.g., Matsuzawa, 2001) and are known to trade services, such as grooming and agonistic support, in the wild (e.g., Mitani, 2006). Chimpanzees also trade commodities for services in some limited contexts, for instance, trading meat for support (Mitani \& Watts, 2001) or, in a captive context, grooming for food (de Waal, 1997). However, at least in the wild, this ability does not seem to extend to commodity trading. Even food sharing is rare outside of the context of hunts and often occurs in contexts other than barter (Hockings et al., 2007; Silk, 1979; Slocombe \& Newton-Fisher, 2005). Thus, researchers have attempted to understand this behavior through captive studies.

A number of species, including chimpanzees, easily trade tokens with human experimenters (Brosnan \& de Waal, 2004; 2005; Chalmeau \& Peignot, 1998; Chen, Lakshminarayanan, \& Santos, 2006; Westergaard, Liv, Rocca, Cleveland, \& Suomi, 
2004). Chimpanzees are sensitive to the cues of the experimenter, for instance responding to begging gestures by increasing rate of return of tokens (Hyatt \& Hopkins, 1998). Chimpanzees also associate value with tokens, with their use of tokens in exchange reflecting a preference for higher-valued tokens (Brosnan \& de Waal, 2005). Token exchange has also effectively been used as a tool to understand other behaviors in chimpanzees (Brosnan, Schiff, \& de Waal, 2005) and as a means of providing reward (Sousa \& Matsuzawa, 2001).

Chimpanzees also will trade items that are useful to them for other items. The first study to investigate this behavior demonstrated that chimpanzees were not only capable of such trading, but intelligent in their understanding of how it worked (Lefebvre, 1982; Lefebvre \& Hewitt, 1986). Juvenile chimpanzees were given pieces of food that could be exchanged with the experimenter for another piece of food. The experimenter would accept any size morsel for the trade, and chimpanzees quickly began consuming most of their food, then trading very tiny morsels of the food, or even a daub of saliva, back to the experimenter for the other food. Subjects traded foods they did not like for those that they did, and they were much more likely to trade foods that were disparate in value than those that were closer in value. This attention to food value is consistent with what is known about food sharing behavior in chimpanzees (Nissen \& Crawford, 1936; Silk, 1979). A recent study replicated these results with adult chimpanzees, although in this case the subjects were given 30 pieces of food that could be exchanged one-for-one, and partial pieces were not accepted. Again, chimpanzees only traded for preferred foods and, as would be expected, rarely traded when foods were close in value. Such behavior potentially represents a trade-off between the gains of trade on the one hand and the risks 
inherent in giving up an item on the other (Brosnan, Grady, Lambeth, Schapiro, \& Beran, 2008). As with token exchange, food exchange has been used to investigate other phenomena in chimpanzees (Brosnan et al., 2007; Dufour, Sterck, Pele, \& Thierry, 2007) In virtually all studies of exchange, subjects are trading with a human experimenter, who clearly has a different position in the social hierarchy than would a conspecific. Moreover, in captive studies the experimenter also typically operates as a moderator or facilitator of behavioral responses, rather than as a passive participant. Only a few examples of chimpanzee-to-chimpanzee exchange exist in the literature (e.g., Paquette, 1992), as this behavior occurs remarkably rarely as compared to situations in which the exchange partner is human (Lefebvre \& Hewitt, 1986). In the one study explicitly investigating trade between chimpanzees, two language trained juvenile males used their symbol system to direct each other towards objects that they needed to obtain food rewards. Although initially the experimenters also rewarded the chimpanzee who correctly gave the tool, this was eventually stopped and the chimpanzee who obtained the tool shared food with the tool provider on his own. The chimpanzees also were able to switch their roles between requestor and provider (Savage-Rumbaugh, Rumbaugh, \& Boysen, 1978). Lefebvre (1982) has also documented a case of two chimpanzees spontaneously exchanging food when each got the other's preferred food - although this occurrence was not repeated. These interactions do, however, indicate that exchange or barter between individuals is within the range of skills for chimpanzees.

In our study, we tested the ability of chimpanzees to barter with each other. Although human barter can emerge spontaneously between interacting individuals in experimental situations (Crockett et al., in press), we set up an experimental situation that 
would both facilitate barter and allow us a great deal of control. To avoid the difficulty of getting a chimpanzee to forfeit an object with immediate use value, such as food, to another chimpanzee, we used chimpanzees that had been trained in a symbol language since infancy (Rumbaugh, 1977; Rumbaugh \& Washburn, 2003; Savage-Rumbaugh, 1986). Chimpanzees were required to exchange tokens that consisted of symbols that were known to be meaningful to both individuals within a pair. Although in many of these situations the tokens did not have immediate use value (i.e., they could not be used to obtain food from the experimenter immediately), all had been and were used by these chimpanzees to obtain food from the experimenter during the course of the experiments. Our goal was to determine whether chimpanzees would spontaneously trade tokens that they possessed to another chimpanzee that could use those tokens and, if not, whether experience with the gains of barter would encourage such behavior to develop.

We presented the subjects with a total of three experiments, each with 3 to 5 different phases. The purpose of the first experiment was to determine whether subjects would accurately trade lexigram tokens to a human experimenter for their corresponding food item, even when tokens were present for foods that were no longer available, or were not available to the subject. The second experiment consisted of four phases, designed to determine whether subjects would trade tokens which they could not use to partners, who could then exchange them for food. Although subjects had limited success, the results did not indicate an ability to barter. Thus, the third experiment was designed to determine which aspects of barter were difficult for the chimpanzees, in an effort to determine why bartering was so difficult for them to achieve. 
Methods

Participants. Four chimpanzees housed at the Language Research Center of Georgia State University were used in this study. Three of these subjects were specifically chosen because three of them had undergone extensive language training using lexigrams, symbols representing words, since infancy. These chimpanzees were Lana (female, 37 years of age), Sherman (male, 34 years of age), and Panzee (female, 22 years of age). The fourth chimpanzee, Mercury (male, 21 years of age), had been raised in the same cognitively enriching environment, but he had not received language training. He was used in initial studies as a control to verify that a meaningful understanding of the tokens' symbols was required in order to complete the task.

All four chimpanzees were highly experienced in a variety of different cognitive tests, including exchange tests (Brosnan et al., 2008), delay of gratification tests (Beran \& Evans, 2006), and tests in which they made judgments of food quantities (Beran \& Beran, 2004). All chimpanzees lived together in a social group in a building that offered indoor and outdoor access, although they were separated for daily test sessions into individual test areas. Water was available continuously, and neither food nor water was ever restricted for the purposes of testing.

All subjects had been trained to exchange prior to the study and had participated in a study of experimental exchange with a human experimenter in the 6 months leading up to this study (Brosnan et al., 2008). One subject, Sherman, had previously completed a series of exchange studies with another male, as described above (see Introduction; Savage-Rumbaugh et al., 1978). Thus, even in the initial studies examining spontaneous exchange, it must be recognized that Sherman had previous testing experiences that had 
taught him the value of exchanging objects with another chimpanzee. The other three subjects, Lana, Mercury and Panzee, had no experience with exchanging tokens in test situations beyond that of the recently completed study in which they exchanged only with a human experimenter (Brosnan et al., 2008).

Subjects were always tested in the same pairs. Because the two females did not always interact well with each other, when subjects were paired together, pairs were always either Sherman/Panzee or Sherman/Lana (Mercury did not participate in studies in which chimpanzees were paired because of his lack of symbol competence). Thus, Sherman participated in more trials than either of the two females. The chimpanzees were tested in adjacent runs that were separated by cage mesh that allowed visual access to their partners. These runs were 6 to 8 feet wide and 10 to 12 feet deep.

Apparatus. The same tokens were used for all of the following studies. Eight foods (M\&M chocolate candies, grapes, apple pieces, orange pieces, carrot pieces, banana pieces, bread pieces, and sweet potato pieces) were chosen for this study. Fruits and vegetables were cut into cubed pieces that were approximately $1 / 2$ inch on each side. Each food was linked with a specific symbol, or lexigram, with which the chimpanzees had had extensive experience since infancy.

Symbols were laminated and glued to each side of a lexan square that was 1 inch by 1 inch by $1 / 2$ inch to make double-sided tokens. Tokens were water-proof, so that the symbols embossed on each side did not fade.

Operational Definitions. One difficulty in studying a behavior in nonhuman animals that is common in humans is the ease with which behaviors may be overinterpreted in light of what we, as humans, experience. In this particular study, we have 
chosen words to indicate specific behaviors, which we here operationally define. For this study, we consider exchange to be the giving of a token to a human experimenter. Trade is the transfer of a token from one chimpanzee to another.

Data Analysis. All data were collected by an observer present during the experiments. Due to the small sample size and atypical experiences of these subjects, statistics are used only to determine whether individual responses differ from chance levels of responding; statistics are not meant to extrapolate from this sample to the larger population of chimpanzees. All data analysis was done using non-parametric statistics (statistic used is indicated). Due to the small sample size, chi-squared tests are performed using the Likelihood ratio (rather than the Pearson chi-square), which is more accurate for smaller samples (Field, 2005). In several cases the expected values are below 5. For binomial tests, all comparisons are made to chance, which is $50 \%$ in all cases. Exact tests are used for all sample sizes under 30. All statistics are two-tailed.

Design and Procedure. The first series of studies were designed to verify that the chimpanzees understood the differential value of the lexigram symbols as a function of their meaning, understood that the tokens could be used to obtain food items, and understood that the tokens could only be used to obtain food items which were present and designated as theirs.

Phase 1. Will subjects trade lexigram tokens for food?

Subjects were given 15 tokens at the start of each trial, with 5 identical tokens for each of 3 different food types. (In later trials, we increased the number of tokens to 20 - 5 tokens each representing 4 different food types - to verify that their performance remained the same with the addition of another token/food). These tokens were 
presented in a shallow bowl that was placed under the cage mesh so that the chimpanzees could access all of the tokens at the same time. Subjects could return the lexigrams to an experimenter, for which they would receive a food item that corresponded to the lexigram symbol on the token. To investigate if subjects had stable preferences that directed their token return behavior, we analyzed the order in which the subjects chose to return the tokens. Subjects had strong preferences among the foods used, and these preferences had been firmly established prior to the outset of the experiment using a forced-choice preference test (e.g., Brosnan \& de Waal, 2004). Therefore, if from the very first trial subjects returned several tokens of the same type together or habitually returned tokens in the same order (especially if those tokens were returned in an order that matched the known preferences for the food items) we could infer that the chimpanzees associated particular tokens with specific food items, most likely on the basis of the meaning of the lexigrams embossed on those tokens. Random order of return would indicate that subjects may not understand the contingency between which token they returned and the food they received.

We tested all four chimpanzees, including Mercury as a control subject, as he had no training in using or understanding lexigrams. In this way we could determine whether the other three chimpanzees’ prior experience with lexigrams affected their ability to use the tokens, or whether this information could be easily obtained through interactions with the tokens (i.e., rapid associative learning). Given the many different ways that three of the four chimpanzees (Lana, Sherman, and Panzee) have used lexigram stimuli in previous tests (e.g., Beran, Savage-Rumbaugh, Pate, \& Rumbaugh, 1999; Menzel, 1999; Savage-Rumbaugh, 1986), we expected that lexigram exchanges would occur in an order 
predicted by known preferences among the food types. We expected that Mercury would not perform in this manner. In fact, Mercury received only 10 sessions (rather than 12), seven with 15 tokens and three with 20 tokens, because he became increasingly disinterested in the task and began refusing to participate, probably as a result of frustration at not being able to obtain the preferred food items that he saw.

Results. Subjects varied in how ordered their choices were in the token exchange. Sherman was the least random in his choices. He was much more likely to return a preponderance of higher-value tokens (as opposed to lower-value tokens) in the first 5 exchanges in a session (e.g. at least 3 of the 5 exchanges; $\chi^{2}$ comparing the number of high-value to low-value tokens exchanged in these first 5 exchanges: $\chi^{2}=53.46, \mathrm{df}=2$, $\mathrm{N}=42, p<0.001$ ), and he became more likely to return tokens in clusters of 5 (the maximum number of any one type of token available; no clusters in first 2 sessions and between 1 and 4 clusters in subsequent sessions) over the course of the sessions (correlating number of clusters with session number: Spearman's rho $=0.745, p=.002$ ). Panzee was similarly likely to return higher value tokens first $\left(\chi^{2}=25.94, \mathrm{df}=2, \mathrm{~N}=42\right.$, $p<0.001$ ), but she did not increase her tendency to return similar tokens in clusters over the course of trials (no clusters in first 2 sessions and between 1 and 4 clusters in subsequent sessions; Spearman's rho $=-0.314, p=0.275$ ).

Lana's behavior was different from that of the other two language-trained subjects. Whereas the other two virtually always returned the tokens representing their favorite foods first (12 of 12 sessions for Sherman and 10 of 12 sessions for Panzee), Lana was equally likely to start with high value and low value foods (she returned tokens for highest value foods first 4 times and returned tokens for least favored food first 5 
times; $\chi^{2}=2.89, \mathrm{df}=2, \mathrm{~N}=42, p=0.235$ ). Although she did return tokens in clusters, she did so less frequently than the other two chimpanzees, and she did not increase her tendency to return tokens in clusters over the course of the sessions (no clusters in first 2 sessions and between 0 and 2 clusters in subsequent sessions; spearman's rho $=-0.108, p$ $=0.713)$. This indicated that Lana's token exchanges did not match her preferences for the food types, and this was problematic for the remainder of the phases in this experiment. Therefore, we chose not to include her in those tests that relied upon a stable order of return for analysis.

Our control subject, Mercury, who was not language trained, preferentially returned high-value tokens only once $\left(\chi^{2}=2.267, \mathrm{df}=2, \mathrm{~N}=30, p=0.322\right)$. Mercury never returned tokens in clusters of 5.

Phase 2. Will subjects limit their return of tokens to foods which are available?

Due to performance in Phase 1, only two subjects (Sherman and Panzee) proceeded to this study. Subjects began each session with a cache of food, which could not be accessed. Each cache was placed in a clear Tupperware bin (approximately 8 inches deep and 12 inches wide) with the subject's picture and lexigram "name” glued to the front to act as cues to which container was going to be used with which chimpanzee. In case subjects did not understand the meaning of his or her picture or his or her lexigram, bins were always placed in the same location for each subject, and each session began with the experimenter calling the chimpanzee over to its bin. Each bin contained a varying number of items, a varying number of food types, and a varying number of each type of food so that the subject could not learn to predict how much or what types of food remained in the bin. 
To obtain the foods in the bins, subjects had to move to an adjacent cage to choose a token from a second experimenter, and they then had to take that token to the first experimenter to receive a reward. The second experimenter laid out on a board one token representing each of the available foods and slid the board under the fence so that the chimpanzee could access it. Each chimpanzee was allowed to choose only one token from the board; if the subject chose more than one token, the board was withdrawn and the selection process was repeated (without any reward) until only a single token was selected. Choices of more than one token rarely happened. After each selection, the second experimenter would replenish the board to include one of each token, shuffle the tokens and make it available to the chimpanzees again. All tokens were available on every trial, regardless of the foods left in the bin. The second experimenter did not know whether the subject received a reward on each trial and, since there were an unpredictable number of food items (see above), could not inadvertently cue the chimpanzee. The first experimenter did not know what token was chosen on each trial until the chimpanzee brought it to the bin for the exchange. If the subject chose a token representing a food that was no longer available, it did not receive any reward but was allowed to continue the session. Subjects had to remember from their previous visit to the bin what foods were still available. Subjects were allowed to keep retrieving tokens until they chose to stop participating. On some occasions, this occurred before the foods were all collected. On other occasions, the subjects brought tokens to an empty food bin. Subjects received 5 sessions; each session consisted of the presentation of a full food bin and the resultant exchanges. Only one session was done per day with each chimpanzee. 
To evaluate the chimpanzees' understanding of the task, we used the same measures as in Phase 1, as well as evaluating how often subjects selected tokens for which corresponding foods were no longer available and whether or not subjects stopped when their bin was depleted. Although the number of items varied, subjects always had access to between 15 and 20 items. Experimenter error rendered the first 5 trials of Sherman's third session unusable (he was accidentally given a token for which he had no corresponding food and not given the token for his most highly valued food item). These 5 trials were repeated.

Results. When he had to determine for himself which tokens to return for foods, Sherman was again very ordered in his exchange. He returned all 5 tokens for his favorite food before returning any other token in all 5 sessions $\left(\chi^{2}=21.270, \mathrm{df}=2, \mathrm{~N}=\right.$ 18, $p<0.001)$. Panzee did not show this pattern $\left(\chi^{2}=3.278, \mathrm{df}=2, \mathrm{~N}=20, p=0.351\right)$. Whereas Sherman typically returned tokens representing the same foods in clusters (returned 5 in a row 13 times, remaining returns in clusters of 4 tokens), Panzee did so only three times, returning tokens in clusters of 2-4. Moreover, out of the 90 completed exchanges (10 were missing due to both the aforementioned experimenter error and disinterest causing Sherman to stop exchanging before he was finished on some sessions), Sherman made only 4 mistakes, returning a token for which a food was no longer available (4.2\%), while Panzee made 18 mistakes out of 100 completed exchanges (18\%). Sherman also never brought tokens to the experimenter after his food bin was empty, while Panzee brought up to 3 additional tokens after the food bin was empty on each of the first 3 sessions.

Phase 3. Will subjects restrict their barter to foods that are available for them? 
Finally, we tested whether Panzee and Sherman understood that they were only able to obtain foods that were available for them, even though other foods (for their partner) were visible. This study was similar to Phase 2, except that the subjects were tested in a pair. This necessitated several changes. First, two containers of food were visible at all times. To reduce confusion, each individual's food bin was always placed in exactly the same location. Second, subjects were now required to alternate turns. The third change was that the token board now contained all of the tokens for both chimpanzees. Thus, there were some tokens on the board representing foods which only one subject would receive. Two to three foods were present in common in both chimpanzees' bins, and each subject had an additional two to three foods which were unique and only present in their bin. The number of different food types and the number of foods of each type varied between individuals and sessions, although both subjects had the same total number of items within the same session. This variability was instituted so that subjects could not rely on tokens their partner chose to obtain their own food items. To complete the task, each chimpanzee had to remember the contents of its own food bin while ignoring the food consumed by the partner (and the tokens relevant to the foods consumed by the partner), move to a separate location within the laboratory and retrieve an appropriate token, and return to exchange with the experimenter. Tokens were always replenished and shuffled between every trial. Subjects received 15 sessions.

Analysis was similar to Phase 2, except we additionally investigated whether subjects chose tokens for food in the partner's bin but not in their own bin.

Results. Sherman continued to outperform Panzee when they worked together. In total, Sherman returned tokens correctly 245 times and incorrectly 33 times (88\% of 
exchanges correct). Panzee returned tokens correctly 222 times and incorrectly 70 times (76\% of exchanges correct). Note that they completed a different number of trials because they often ceased participating after their favored foods were gone. To control for this, all statistics are based on the proportion correct, rather than the absolute number. Both subjects seemed to understand the task from the outset, as neither improved (i.e., returned more tokens correctly) over the course of the 15 sessions (Sherman: Spearman's rho $=0.393, p=0.148$; Panzee: Spearman's rho $=0.329, p=0.230)$. However, Sherman was more likely to return a correct token than Panzee $(\chi 2=14.01$, $\mathrm{df}=2, \mathrm{~N}=500, \mathrm{p}<$ .01). Sherman also was error-free on 5 of 15 sessions (33\%) while Panzee made errors on all 15 sessions. Two types of errors could be committed: returning a token for a food which only the partner still had or returning a token for a food which the subject had earlier in the trial but had already depleted. Panzee committed a larger percentage of errors of the former type, asking for a food which belonged to Sherman, while Sherman did not commit more of one error type than the other (Sherman, 61\%, p =0.296; Panzee, 79\%, $p<0.001$; binomial tests).

\section{Discussion}

Two of our subjects, Sherman and Panzee, clearly understood the use of lexigrambased tokens to obtain food rewards from human experimenters. Both chimpanzees learned to request only foods that were available for them and neither frequently requested the foods for the other chimpanzee or the foods already depleted from their own bins. However, Sherman performed at a higher level than Panzee throughout the tests. Sherman's superior abilities to Panzee were likely due to his early experience trading tools with another male chimpanzee (Savage-Rumbaugh et al., 1978). 
The other two subjects did not use these tokens to obtain foods in the same way. Mercury, our control subject who had not been language trained, did not learn to trade meaningfully even after 10 sessions, indicating that he had not learned which tokens were to be matched with which foods. This suggests that paired associations of specific tokens and foods were not easily learned during the experiment. Instead, facile performance required the subjects’ previous experience with the lexigrams. Lana was more puzzling. Other experiments have confirmed that she knows the referents for these particular lexigrams (Beran et al., 1998). As with Sherman and Panzee, she tended to return tokens in clusters, but did not return tokens in an order related to her food preferences, as would be expected if she understood the meaning of the tokens. Perhaps Lana simply did not care which foods she received at which time (ultimately she received all foods which were available), or perhaps she was simply matching tokens, returning those which were the same as the last token returned. Although her lack of consistency would have rendered results in Phase 2 and Phase 3 of this experiment difficult to interpret, we chose to include Lana in some later testing, in case her results were due to the first possibility.

\section{Experiment 2}

\section{Methods}

Participants and Apparatus. These were the same as in Experiment 1, except that Mercury was no longer included in testing.

Design and Procedure. As it had been verified that some subjects understood the use of the tokens, the next series of studies were designed to see if chimpanzees would spontaneously give tokens to each other. Specifically, we created situations in which 
trade between chimpanzees would allow each partner to obtain food rewards that were not available without barter.

Phase 1. Do chimpanzees recognize when they could maximize rewards by trading tokens with a partner?

For this study, two chimpanzees (Sherman and Panzee) were paired and sat in adjoining test areas, separated by mesh. Lana was not included in this study because we wanted to provide the best case for potential spontaneous barter by chimpanzees, and Lana's difficulties in Experiment 1 suggested that she was not the best participant for this phase of the experiment. Sherman and Panzee could pass tokens underneath the mesh to each other, but they could not take tokens from the other. For each trial, each chimpanzee was initially shown one of two different foods, which was placed in a shallow, clear bowl (6 inches in diameter) in front of them by Experimenter 1. Experimenter 2 sat between the two subjects and did not know which subject got which food (the foods were located behind him and out of his view). Next, Experimenter 1 gave Experimenter 2 a token to pass to each chimpanzee. Half of the time, the token that the chimpanzee received matched that chimpanzee's food item, while half of the time the token that the chimpanzee received matched their partner's food item. This varied randomly so neither Experimenter 2 nor the chimpanzees could predict the outcome. Finally, Experimenter 2 offered the designated token to each chimpanzee, and then the focal animal (this alternated, and was the chimpanzee on which Experimenter 2 focused) on a trial was allowed to exchange that token back with Experimenter 2 or trade his or her token to his or her partner. Subjects were not given encouragement either way, and Experimenter 2 always accepted a token if offered (Experimenter 2 did not know which 
action would bring the chimpanzee a reward and therefore could not cue the chimpanzee to exchange or return specific tokens). If either chimpanzee chose to give his or her token to the partner, the second chimpanzee was required to give a token back before either was allowed to exchange with Experimenter 1. This was enforced by the experimenter refusing to accept tokens until the second chimpanzee had given a token to the first. Note that this does not mean that the second chimpanzee was forced to make a 'bad' trade, as that subject could choose to return either the token it had just been given by its partner or the token it had been given by the experimenter. Each session consisted of 12-17 trials (the session ended when either subject left the testing area, so the total number of trials varied) and each pair participated in 8 sessions. Sherman and Panzee alternated making the first decision (exchange or trade) in a trial, which was indicated to them by which chimpanzee was the focus of Experimenter 2's attention.

We analyzed whether the subjects chose to do the correct thing by trading a token that did not match their own food item with their partner or exchanging a token that did match their own food item with the experimenter, and whether the number of correct choices increased over the course of sessions or trials. Also, there were some cases in which a chimpanzee gave the 'wrong' token to their partner, so we further investigated whether, in this case, the partner returned that token, keeping the one that it needed (in essence, did that chimpanzee correct the error made by its partner?). We finally investigated which type of error was most common, choosing to trade a token to the partner when this was not warranted or choosing to exchange a token with Experimenter 2 even though the token did not match the food reward that was available. 
Results. Both subjects performed the correct action (trade token with partner or keep token) at levels higher than expected by chance (Sherman: 74.89\% accurate; Panzee: 73.34\% accurate; $p<0.001$ for both, binomial test). Neither subject improved in accuracy over the course of the eight sessions (Sherman: Spearman's rho $=0.587, p=$ 0.126; Panzee: Spearman's rho $=0.703, p=0.078$ ). Of situations in which their partner made a mistake, giving the partner a token when they should not have, Sherman returned the token to Panzee on 75\% of trials (6 of 8) and Panzee returned it to Sherman on 67\% of trials (2 of 3).

Phase 2. Do chimpanzees succeed more often when they do not have to decide whether to trade their only token, but instead must decide which of two tokens to trade?

To verify that subjects continued to understand the association between lexigram tokens and food, prior to each session in this experiment, each subject was given a series of trials in which they were given 2 tokens and had to return the one that matched the proffered food to receive it. All 3 subjects (Lana, Sherman, and Panzee) succeeded on > $90 \%$ of trials (see results for details). Because Lana now was able to succeed in this prerequisite task for the formal experimental phase, she was returned to the study at this point and worked as a partner with Sherman.

In the previous study, the interaction was more of a matching game than true barter. Subjects had only to trade with their partner if their food did not match their token and to exchange with the experimenter if their food did match their token. To bring this closer to barter, we next designed a study in which subjects were required on every trial to trade a token with their partner before they could exchange a token with the experimenter, but each subject had to decide which of two tokens in their possession to 
trade. The set-up for this test was similar to that of Phase 1 except that subjects were given two tokens, one of which was useless to both chimpanzees and one of which was useful to either the subject or its partner. Again, Experimenter 2 did not know whether the tokens matched the subjects’ available food. Prior to being allowed to exchange, a subject had to give one token of his or her choice to his or her partner, who then had to give one token to the subject. We analyzed whether each subject chose to trade the correct token to its partner and whether the subject corrected its partner's mistakes.

Results. All subjects returned tokens that matched the proffered food reward in the preliminary trials (Sherman: 91\% accurate, $p=0.012$; Panzee: 91\% accurate, $p=$ 0.012, Lana: $92 \%$ accurate, $p=0.003$; binomial tests), indicating that they continued to understand that tokens could only be used to acquire available foods. Note that this was the first demonstration that Lana understood the task, and the first time all subjects were similarly successful. We do not know why Lana suddenly was successful on a task that was functionally very similar to that on which she had earlier shown little competence. Perhaps something changed her motivation for the task, but we do not have empirical data to answer this question.

In Phase 2 test trials, subjects had to determine which of two tokens to trade, and responses were highly variable. Sherman traded the correct token to his partner in every situation (100\% correct trades, $p<0.001$, binomial test). Lana performed at chance, trading the correct token to her partner about half the time (44\% correct trades, $p=0.815$, binomial test). Panzee, on the other hand, was marginally more likely to trade the incorrect token to her partner (25\% correct trades, $p=0.077$, binomial test). Although we cannot definitively say why this occurred, it should be noted that, subjectively, it 
appeared that Panzee’s first movement was to collect the token which matched the food in front of her, then when she was required to trade, she failed to switch this token that was useful to her for the other one. It may be that this was an impulse control problem, in which Panzee was unable to refrain from acting on the token she knew could generate rewards even when she had to trade that token away.

None of the chimpanzees showed any improvement in performance over time (of course, Sherman’s behavior did not leave room for improvement; he was already maximizing rewards). It is possible that the subjects did not become better with experience because even with the high percentage of incorrect trades, subjects continued to be rewarded more often than chance (Sherman: $80 \%$ of trials rewarded, $p<0.001$, Panzee: $68 \%$ of trials rewarded, $p=0.023$; Lana: $74 \%$ of trials rewarded, $p=0.002$ ). This is because trading alternated between subjects, and both females were paired with Sherman, who was very good at the task. Thus, they were rewarded on each of Sherman's trials, as well as their own trials that they got correct. This also explains why Sherman was rewarded on only $80 \%$ of trials even though he was $100 \%$ accurate (because of incorrect trades by his partners). Note that in this experiment, since subjects were given tokens sequentially, mistakes could not be corrected by the partner.

Phase 3. Do chimpanzees succeed more often when all of the available tokens are useful to either them or their partner?

During the previous task, subjects could have been confused by the presence of tokens that were not useful to either them or their partner, a novel situation in this experiment. Thus, in this phase, subjects were again given 2 tokens, but one was valuable only to the subject and one was valuable only to the partner (in terms of 
matching available food rewards). Chimpanzees had to give a token to their partner prior to exchanging a token with the experimenter. Therefore, the chimpanzees needed to decide which token to keep to match to their own food item, and then trade away the other token in their possession. Chimpanzees again alternated trials but, to avoid confusion, only one individual was given his or her tokens at a time. Again, Experimenter 2 did not know which token each chimpanzee needed to give to its partner and which it should keep. For analysis, we investigated whether subjects traded the correct token and whether the subjects corrected their partners’ mistakes (by returning a token which did not match their reward). As in Phase 2, each session also started with trials in which the chimpanzees were given 2 tokens and had to return the one that matched a proffered food to receive it.

Results. In pre-test sessions, all subjects continued to correctly choose the token that matched the proffered reward (all subjects made 100\% correct choices; Sherman: $p=$ 0.002; Panzee: $p=0.002$; Lana: $p=0.008$, binomial tests).

In the formal test, subjects had to choose which of two tokens to keep, one of which was valuable to them and one of which was valuable to their partner. Sherman and Lana both gave to their partner the token that their partner could use (which is also the one that they could not use; Sherman: $98 \%, p<0.001$; Lana: $72 \%, p=0.020$; binomial tests). Panzee, however, almost always chose to give to her partner the token that she herself needed to obtain food ( $6 \%$ correct, $p=0.001)$, perhaps indicating that she again was fixated on the token she needed. Neither Panzee nor Lana ever corrected errors by their partners, by returning the token that the partner needed. Sherman corrected only one $(0.07 \%)$ of Panzee's errors. However, note that in this case, subjects gained nothing 
from correcting partners' mistakes, which may have led to the low occurrence of this behavior.

Phase 4. Will chimpanzees choose a token required by their partner when multiple tokens are available to be traded?

In the previous task, subjects could succeed simply by trading the token that they did not require. Thus, we did a final barter task that was identical to Phase 3, except that the subject was given 3 tokens, one that matched their food, one that matched their partner's food, and one foil, which did not match any available food. For analysis, we investigated whether subjects were more likely to give their partner the token their partner needed, or the foil. Only Sherman and Lana were used because they were the only two chimpanzees who were above chance in Phase 3.

Results. In this task, subjects had to choose whether to give the partner the token that the partner needed or to give the foil; neither subject consistently gave to the partner the token that matched the partner's reward (Sherman: 29\% correct, $p=0.070$; Lana: 0\% correct, $p<0.001$, binomial tests). However, although this behavior may seem to be unexpectedly poor (e.g., as if subjects were trying to give their partner the incorrect token), this reflects only responses in which a single token was traded to the partner. Both Lana and Sherman adopted a simpler strategy of trading both tokens to the partner, who then retrieved the correct one for exchange with the experimenter. Lana behaved in this way virtually all of the time (95\%, $p<0.001$, binomial test), while Sherman did so occasionally (33\%, $p=0.189$ ). Thus, if we include trials in which both tokens were given to the partner in the category of correct trades, Lana’s performance becomes significantly different from chance (95\% correct; $p<0.001)$, although Sherman’s choices 
were still random $(62 \%$ correct, $p=0.383)$. However, it is unclear whether this latter circumstance represents a desire to help the partner or represents the chimpanzees’ (mis)understanding of how the game was to be played. Note that neither subject ever traded away tokens that they needed to obtain their own foods. Because of this, neither subject had the opportunity to correct their partner's errors.

\section{Discussion}

Two of the three subjects did learn to perform barter-like behavior, in which one chimpanzee traded to its partner the tokens that matched the partner's current needs, although one subject, Sherman, consistently performed better than Lana, the other successful chimpanzee. However, it does not appear that the subjects actually understood the behavior as barter; in every case performance can be explained by matching (e.g., the subject needed to give the partner the token that he or she did not need) and in no case was an understanding of the partner's needs demonstrated. In the critical task (Phase 4), in which subjects needed to pay attention to the partner's needs in order to give the correct token, there was no evidence that either subject was doing so. None of this can be explained by a lack of association between the tokens and the foods, as all subjects continued to show near perfect understanding of the tokens' meanings in control trials, virtually always trading the token matching the available food reward with the experimenter.

We thus conclude that even chimpanzees with extensive cognitive and symbolic experience do not spontaneously and appropriately trade tokens with other chimpanzees, even when it might be in their interest to do so (e.g., their partner has a token they require). Note, too, that this is the simplest form of barter because the tokens that the 
subjects can exchange have no current use value for them. The only risk is in giving up something to the partner without receiving anything in return, but there is no risk of losing a commodity as would be true in more costly bartering situations. Thus, the fact that we do not see barter in this situation indicates that barter is quite difficult for chimpanzees. However, these results do not indicate what it is about barter (or our experiments) that causes difficulty for the chimpanzees. Thus, we designed a final series of studies to investigate each of the behaviors required to complete this task individually, to try to determine at what point the understanding of barter breaks down for these chimpanzees.

\section{Experiment 3}

\section{Methods}

Participants and Apparatus. These were the same as in Experiment 2.

Design and Procedure. Even after several iterations of the barter paradigm designed to elicit trade between chimpanzees, there was little evidence that any of the subjects understood the task as barter. Thus, we designed a final series of studies that explicitly modeled the behaviors required for bartering objects between apes. In this way, we could uncover possible aspects of the study or task that made exchange difficult, and give the chimpanzees every opportunity to understand trade.

Phase 1. Will chimpanzees pass tokens to a partner for their own gain?

One possible explanation for some of the previous results is that chimpanzees did not realize that passing a token to their partner would allow their partner to obtain the food rewards which were present in front of them. Thus, we designed the current study 
to draw the subjects' attention to their partners' gains, and to increase the benefit to the subject for paying attention to their partner's gains.

For this study, a larger food reward than in the previous experiments (2 large pieces of banana or $8 \mathrm{M} \& \mathrm{M}$ candies) was divided and placed in front of the partner. The subject was presented with 2 tokens on a board by Experimenter 1 (as in previous studies), one of which matched the large quantity of the single food type in front of the partner and one of which did not. Experimenter 2 then asked the partner for an exchange, using the standard gesture. To get the food, the subject had to slide a single token to its partner, who then could exchange it with Experimenter 2. If the token that was returned matched the food, the experimenter then gave each chimpanzee an equal amount of the food. If the chimpanzee passed the wrong token to the partner, neither animal received anything and there was a time out period of 10 seconds before the next trial. Note that as with the previous experiments, subjects' initial action was a trade to the partner, so this was a familiar activity. However, in this case it was the trade that generated their reward, not an exchange with the experimenter, which was designed to emphasize the gains to be had from giving the correct token to the partner.

Subjects did not alternate during these sessions; each subject completed 12 trials, passing tokens to the same partner. Initially, verbal prompting was used as needed to encourage the subjects to pass one token to the partner. In these cases, Experimenter 2 verbally instructed subjects to "give a token to (partner name)" while pointing between the two tokens, but at no point did this experimenter indicate that one token should be given instead of another (and, again, this experimenter did not know what food was available as the reward). For analysis, we examined the number of correct trials (in 
which the subject passed the correct token to the partner) and the number of trials that required prompting. Subjects completed 2 sessions of 12 trials each.

Results. All subjects gave the token which matched the available food type to their partner in order to receive their half of the food bonanza (percent correct: Sherman 96\%, Panzee 100\%, Lana 88\%; all p’s < 0.001, binomial tests). However, subjects differed on whether they required verbal cuing to give a token to the partner. (Verbal cuing was not directed at any token.) Sherman and Panzee required little prompting (Sherman: 8\%; Panzee: 17\%) while Lana required a great deal of prompting (63\%).

Phase 2. Will chimpanzees discard a useless token before exchanging the correct token with an experimenter?

In previous studies, subjects may have been disinclined to pass tokens that were worth a desirable food item to their partners. Thus, this study was designed to see if subjects would be able to discard a token into an adjacent cage with no other chimpanzee present to receive it. The protocol was identical to that of Phase 2 in Experiment 2, except that no partner was in the other run. Thus, subjects traded 12 times in succession, rather than alternating between individuals, as in the previous study. Subjects were given two tokens by Experimenter 2, one of which matched a food in front of them (placed by Experimenter 1) and one of which matched no visible food. They were required to discard a token into the adjoining cage, following which they could exchange the remaining token with Experimenter 2. If it matched the available food reward, they received it. At the beginning of the session, verbal prompting was occasionally needed to indicate to the subject that they needed to first discard a token to the other cage, but at no time was a specific token indicated (and controls against potential cuing remained in 
place, as the Experimenter 2 did not know which food was available). For this study we analyzed whether subjects chose to discard the appropriate token and whether verbal prompting was required. Subjects completed 4 sessions of 12 trials each.

Results. All subjects discarded the token that they did not need into the empty enclosure (percent correct: Sherman 88\%, Panzee 85\%, Lana 87\%; all p’s $<0.001$, binomial tests). In this study, subjects generally did not require verbal prompting to discard a token to the other run (percent trials prompted: Sherman 6\%, Panzee 23\%, Lana $13 \%)$.

Phase 3. Will chimpanzees give a token that is useless to them to their partner? This study expands on Phase 2 by including a partner in the adjacent cage. This allowed us to determine whether the reluctance to give tokens to the partner in the earlier studies was due to not understanding that a token should be discarded (addressed in Phase 2 of this experiment) or not wanting to give a token to the partner. The only difference between this study and Phase 2 of Experiment 2 was that subjects performed all 12 trials in a row, rather than alternating back and forth (as in Phase 2 of the current experiment). This was done to avoid the possibility that something in the nature of the alternation confused the subjects. No verbal cuing was used in this study. We again analyzed whether subjects chose to discard the appropriate token to their partner. Subjects completed 3 sessions of 12 trials each.

Results. All subjects gave to their partner the token that they did not need (percent correct: Sherman 100\%, Panzee 81\%, Lana 94\%; all p’s < 0.001, binomial tests). Only Sherman had the opportunity to correct the mistakes of his partners (Sherman made no mistakes, so Panzee and Lana always received the correct token). However, Sherman 
only corrected mistakes about half of the time (corrected 64\% of errors, or 9 of 14, $p=$ 0.424, binomial test). He received no rewards for correcting his partners' mistakes (given the constraints of this task) and so he may have lacked motivation for such corrections.

Phase 4. Do chimpanzees evaluate whether token exchange is appropriate for a given situation?

Once it was verified that subjects would give partners tokens, the last test examined whether subjects were paying attention to what they and their partner had, or whether they simply learned to always trade a token. This study replicated Phase 1 of Experiment 2, in which subjects received 1 token which $50 \%$ of the time matched their available food and 50\% of the time matched their partner's available food. Thus, subjects had to determine whether or not trade with the partner was appropriate. The only difference between this study and Phase 1 of Experiment 2 was that the chimpanzees did not alternate roles (as subject or partner) between trials, but instead completed 12 trials consecutively. Again, we analyzed whether subjects chose correctly whether or not to trade their token and how partners responded if the subject gave them the 'wrong' token. Each chimpanzee was the focal subject for 4 12-trial sessions.

Results. All subjects made correct decisions about whether to keep a token or exchange a token correctly (percent correct: Sherman 96\%, $p<0.001$; Panzee 73\%, $p=$ 0.002; Lana 83\%, $p<0.001$, binomial tests). Subjects also occasionally corrected errors made by their partner. Sherman made two errors when paired with Panzee, both of which she corrected. Sherman corrected errors at chance levels (37\% of errors corrected, $p=$ 0.359, binomial test) and did not discriminate between partners (he corrected three errors for each). 


\section{Phase 5. Will chimpanzees barter in an unrestricted situation?}

In previous studies, chimpanzees were required to perform an action that appeared to be barter (give a token to their partner) before they could exchange. However, in all of those studies, the barter behavior can be explained by subjects matching their tokens to their available rewards and then discarding non-matching tokens. Thus, in this final experiment, subjects were given 4 tokens, each matching a different food, and Experimenter 1 gave them each two servings of each of two foods (each subject had different foods so that if they traded, they could receive all 4 foods). Subjects were allowed to exchange with Experimenter 2 or trade however they chose. Exchanging out the tokens they had would acquire half of the food, but they required two tokens from their partner to obtain all of the food. Subjects received 2 sessions of 12 trials (each trial was the entire interaction that began with the presentation of the 4 tokens). If subjects truly understood barter, they should trade tokens that did not match their food items and retain tokens that did. Given their performances in Phase 4 and the challenging nature of this study, we paired the two subjects who had performed best, Sherman and Lana, for initial tests. In their test, Sherman and Lana quickly ceased trading altogether (see Results, below), so we chose not to test either with Panzee as they would be entering the test predisposed to not trade, which would presumably have affected her responses.

Results. To determine the frequencies of each behavior, we first calculated how many of their actions with the tokens were exchanges versus trades. The ratio which would have garnered them the most rewards was to complete each behavior half of the time, assuming that they chose correctly when to trade and when to exchange. However, both subjects were much more likely to exchange tokens with the experimenter than to 
trade them with each other (Sherman: 83\% of actions were exchanges; Lana: 79\% of actions were exchanges; both $p<0.001$, binomial tests). Although exchanges were preferred, only Sherman was overall more likely to exchange with the experimenter, rather than trading with his partner on the first move (96\% of first moves in both sessions were exchanges, $p<0.001$, binomial test). Lana traded first in her first session (25\% of first moves were exchanges, $p=0.041$, binomial test), but in her second session she exactly reversed this percentage (75\% of first moves were exchanges, $p=0.146$, binomial test). Both subjects traded more in the first session than in the second, perhaps indicating that the behavior disappeared without reinforcement or enforcement from the experimenter (Sherman: 78\% of trades in first session, $p=0.031$; Lana: $85 \%$ of trades in first session, $p=0.001$, binomial tests). Of course, in previous experiments, subjects were required to trade with each other before exchanging with the experimenter. Thus, increased trading in the first session likely means that the subjects did not yet realize that trading was no longer enforced, rather than that they were more likely to try to help their partner.

Of their successful trades, Sherman typically gave Lana the token which would help her (89\% of trades, $p=0.001$, binomial test), indicating that he was paying attention to what she needed, but Lana was indifferent to what Sherman needed (46\% of trades helped Sherman, $p=0.845$, binomial test). Perhaps not surprisingly, given that they usually simply exchanged all of their tokens with the experimenter, neither subject exchanged correctly overall (Sherman: $60 \%$ of exchanges correct, $p=0.066$; Lana: 56\% of exchanges correct, $p=0.278$, binomial tests; exchanging all tokens with the experimenter with no trading would mean $50 \%$ of exchanges correct). However, it 
appeared that subjects were often first exchanging the two tokens for which they had foods, thus, we examined whether subjects’ first and second exchanges were correct. Both subjects' first exchanges were typically for foods that were available to them (Sherman: 96\%, $\mathrm{p}<0.001$; Lana: 73\%, $p=0.052$, binomial tests), although the second exchanges were not (Sherman: 61\%, $p=0.405$; Lana: 55\%, $p=0.832$, binomial tests). Discussion

None of the chimpanzees had difficulty with the first four phases of this experiment, which included the behaviors that we considered building blocks to trade. Thus, all individuals could identify which token was needed to be traded to the partner, as long as the experimenter was enforcing a specific protocol which required giving a token to the partner. However, when put in an unrestricted situation in which trade was not enforced, trade virtually disappeared by the second session. Instead, subjects usually exchanged all tokens with the experimenter, whether they could obtain foods for them or not. One subject, Sherman, did continue to trade the correct tokens, those his partner needed, when he did trade. Perhaps the breakdown in trade was caused not by inability to understand or lack of property (property in this case was consistently maintained by the experimenter) but by a lack of trust of the partner's performance. It may also be the case that chimpanzees have difficulty viewing this barter situation from multiple perspectives at one time, as would be required to understand both their own needs and the needs of the partner. This would be a critical skill for establishing a valid barter system, especially in the absence of communicative interactions that allowed each partner to indicate what it wanted in the barter.

General Discussion 
In our study, chimpanzees who were already proficient at exchange with a human experimenter did not spontaneously barter tokens with each other in a situation in which barter would have increased their payoffs. However, after a series of studies designed to focus on the constituent behaviors necessary to understand barter, some subjects were able to complete a highly structured barter task, giving each other tokens that the other needed to obtain food. Nonetheless, chimpanzees almost certainly did not understand the behavior in the same way that humans do; when chimpanzees were subsequently given an unstructured barter task, in which they could do what they wished with the tokens and the experimenter did not enforce trade behavior, subjects failed to engage in significant trade behavior. This raises the question of why these intelligent primates seem to be unable to maximize their gains by engaging in trade, after extensive training which highlighted the benefits.

One possibility is that, without active intervention from the experimenter, the risks of trade are too great. Although these are not food deprived animals, for whom the cost of losing a food item is severe, even the inequity induced if the partner were to get more food could be very salient to them (Brosnan et al., 2005). Both subjects were much more likely to trade with their partner in the first of their unrestricted tests, but this behavior disappeared later in the test (Phase 5 of Experiment 3). Perhaps this curtailing was due to the recognition that the experimenter was not intervening to compel their partner to trade (which, in previous studies, typically brought them the token that they needed). Without such enforcement, it may not be worth the risk of giving up a commodity unless the potential gains are quite high (Brosnan et al., 2008). Of course, we do not know what humans would do outside of the extensive protections in place for most 
of our trades, either. Perhaps without the opportunity to recruit support, institutionalized in the form of legal protections, barter would be much less common.

A second possibility is that, as chimpanzees have evolved without any sort of property, beyond behaviors related to possession, barter has not become a part of their repertoire (Brosnan et al., 2008). However, in the current study we instituted a property system that indicated which things 'belonged' to them and which 'belonged' to a partner. All subjects were very good at identifying which foods were theirs and making token selections based on only their own food arrays, indicating that they at least grasped the idea of 'their' property. Thus, unless subjects' understanding of their 'property' changed in the last phase of the last experiment, it is more likely that some other factor, such as the absence of experimenter intervention, led to the change in behavior.

It is possible that, with additional training, chimpanzees could learn to barter effectively with each other. Although the current study represents a sizeable investment in training, it likely pales in comparison to the exposure that humans get before independently entering a market economy. Moreover, human training comes not from an individual of a different species, but from conspecifics, and often from kin or trusted individuals (e.g. parents or older relatives and friends). In support of this, we suspect that Sherman's superior performance, as compared to the females, may be due to his previous exposure to the gains of trade (Savage-Rumbaugh et al., 1978). Of course, with a single subject, this cannot be conclusively demonstrated.

We also believe that barter is facilitated in situations when each partner can effectively communicate its needs and intentions. In this experiment, the partner's needs were assessed solely by the other chimpanzee, because neither animal had a way to 
communicate what it wanted. Perhaps if the chimpanzees could have requested specific tokens from the partner during the trading process, as in earlier studies (SavageRumbaugh et al., 1978), more spontaneous and appropriate trade might have developed over time.

It is possible that barter behavior is particularly difficult for chimpanzees as it requires fairly advanced empathy and other-regarding behavior. While anecdotal evidence for such behavior does exist (i.e. de Waal, 2006), as does experimental evidence for 'helping' behavior (Warneken \& Tomasello, 2006; Warneken, Hare, Melis, Hanus \& Tomasello, 2007), several recent experimental studies have failed to find other-regarding preferences in food contexts in chimpanzees (Silk et al., 2005; Jensen et al., 2006). Note, too, that in previous studies finding empathy, it was often the case that animals were in significant distress which was visually obvious to the partner (e.g., pain; Church, 1959; Wechkin, Masserman \& Terris, 1964; Masserman, Wechkin \& Terris, 1964; Langford et al., 2006). This raises the possibility that the 'need' in the current experiment was insufficiently great, as these subjects were never food deprived and were working for preferred foods, rather than survival. Of course, this may also have been less relevant, as giving a correct token to the partner often advanced the subjects' individual needs. For instance, Panzee consistently gave the wrong token to Sherman, which meant that she did not receive a reward.

Another potential cognitive limitation that may prevent appropriate and adaptive bartering pertains to mental score-keeping (de Waal, 2000). Reliable memory for past interactions with other individuals would be required, including memory for beneficial and non-beneficial interactions with each potential trading partner. Although 
chimpanzees have excellent memories for interactions with peers in a variety of past contexts, those memories may not be as reliable for the specifics of the trade results (e.g., being cheated by a partner) as for other types of interactions (such as grooming bouts, aggressive altercations, or food sharing instances; de Waal, 1989). Recent evidence indicates that chimpanzees do not often succeed in reciprocal situations, another behavior which is related to barter (Melis, Hare, \& Tomasello, 2008; Brosnan et al., in revision).

It is possible that barter evolved first through services. Services, such as grooming or support, cannot typically be stolen and do not need to be stored (Brosnan et al., 2008), making them a relatively cost-free way to trade benefits. In fact, some of the best examples of reciprocal behavior in chimpanzees involve services (Mitani, 2006; de Waal, 1989). Such behavior could be extrapolated to goods once protections were in place to protect goods and recruit support against wrongdoers (which likely requires language for events that occur away from others). This leap from chimpanzees to the ubiquitous barter seen in human societies (Chapman, 1980) may be unique to hominid ancestors.

Barter can provide significant benefits, provided that sufficient protections are in place to keep individuals from losing their goods or being taken advantage of. As these data show, chimpanzees are capable of trading objects with each other. They do so in meaningful ways and even show some of the same biases in trade as are seen in humans (Brosnan et al., 2007). However, they do not use barter behavior to maximize their gains in the same ways as humans in market economies do. Whether this is due to a lack of experience, a lack of enforcement, or a lack of explicit and effective communication is difficult to say. It appears that extensive barter may represent a relatively recent evolutionary development in the hominid lineage. 


\section{References}

Beran, M. J., \& Beran, M. M. (2004). Chimpanzees remember the results of one-by-one addition of food items to sets over extended time periods. Psychological Science, 15, 94-99.

Beran, M. J., \& Evans, T. A. (2006). Maintenance of delay of gratification by four chimpanzees (Pan troglodytes): The effects of delayed reward visibility, experimenter presence, and extended delay intervals. Behavioural Processes, 73, 315-324.

Beran, M. J., Savage-Rumbaugh, E. S., Brakke, K. E., Kelley, J. W., \& Rumbaugh, D. M. (1998). Symbol comprehension and learning: A "vocabulary" test of three chimpanzees (Pan troglodytes). Evolution and Communication, 2, 171-188.

Beran, M. J., Savage-Rumbaugh, E. S., Pate, J. L., \& Rumbaugh, D. M. (1999). Delay of gratification in chimpanzees (Pan troglodytes). Developmental Psychobiology, 34, 119-127.

Berg, J., Dickhaut, H., and McCabe, K. (1995). Trust, reciprocity, and social history. Games and Economic Behavior, 10, 122-142.

Boesch, C., Boesch, H., \& Vigilant, L. (1996). Cooperative hunting in chimpanzees: Kinship or mutualism? In P. Kapeller \& C. P. van Schaik (Eds.), Cooperation in Primates and Humans (pp. 139-150). Berlin: Springer.

Brosnan, S. F., \& de Waal, F. B. M. (2004). A concept of value during experimental exchange in brown capuchin monkeys. Folia Primatologica, 75, 317-330.

Brosnan, S. F., \& de Waal, F. B. M. (2005). A simple ability to barter in chimpanzees, Pan troglodytes. Primates, 46, 173-182. 
Brosnan, S. F., Grady, M., Lambeth, S., Schapiro, S., \& Beran, M. J. (2008). Chimpanzee autarky. PLoS ONE, 3, e1518.

Brosnan, S. F., Jones, O. D., Mareno, M. C., Richardson, A. S., Lambeth, S. P., \& Schapiro, S. J. (2007). Endowment effects in chimpanzees. Current Biology, 17, 1-4.

Brosnan, S. F., Schiff, H. C., \& de Waal, F. B. M. (2005). Tolerance for inequity may increase with social closeness in chimpanzees. Proceedings of the Royal Society of London B, 1560, 253-258.

Brosnan, S. F., Henrich, J., Mareno, M. C., Lambeth, S., Schapiro, S., and Silk, J. B. (in revision). Chimpanzees (Pan troglodytes) do not develop contingent reciprocity in an experimental task. Animal Cognition.

Chalmeau, R., \& Peignot, P. (1998). Exchange of objects between humans and captive western lowland gorillas. Primates, 39, 389-398.

Chapman, A. (1980). Barter as a universal mode of exchange. L'Homme, 20, 33-83.

Chen, M. K., Lakshminarayanan, V., \& Santos, L. R. (2006). How basic are behavioral biases? Evidence from capuchin monkey trading behavior. Journal of Political Economy, 114, 517-537.

Church, R. M. (1959). Emotional reactions of rats to the pain of others. Journal of Comparative and Physiological Psychology, 52, 132-134.

Crockett, S., Smith, V. L, and Wilson, B. J. (in press) Exchange and specialization as a discovery process. Economic Journal.

de Waal, F. B. M. (1997). The chimpanzee's service economy: Food for grooming. Evolution and Human Behavior, 18, 375-386. 
de Waal, F. B. M. (1989). Food-sharing and reciprocal obligations in chimpanzees. Journal of Human Evolution, 18, 433-459.

de Waal, F. B. M. (2000). Attitudinal reciprocity in food sharing among brown capuchin monkeys. Animal Behaviour, 60, 253-261.

de Waal, F. B. M. (2006). Primates and philosophers. Princeton: Princeton University Press.

Dufour, V., Sterck, E. H. M., Pele, M., \& Theirry, B. (2007). Chimpanzee (Pan troglodytes) anticipation of food return: coping with waiting time in an exchange task. Journal of Comparative Psychology, 121, 145-155.

Field, A. (2005). Discovering statistics using SPSS, Second Edition. London: Sage.

Fragaszy, D. M., Visalberghi, E., \& Fedigan, L. M. (2004). The complete capuchin: The biology of the genus Cebus. Cambridge: Cambridge University Press.

Hockings, K. J., Humle, T., Anderson, J. R., Biro, D., Sousa, C., Ohashi, G., Matsuzawa, T. (2007). Chimpanzees share forbidden fruit. PLoS ONE, 2, e886.

Hyatt, C. W., \& Hopkins, W. D. (1998). Interspecies object exchange: Bartering in apes? Behavioural Processes, 42, 177-187.

Jensen, K., Hare, B., Call, J., and Tomasello, M. (2006). What's in it for me? Selfregard precludes altruism and spite in chimpanzees. Proceedings of the Royal Society of London, Series B, 273, 1013-1021.

Langford, D. J., Crager, S. E., Shehzad, Z., Smith, S. B., Sotocinal, S. G., Levenstadt, J. S., Chandra, M .L., Levitin, D. J., Mogil, J. S. (2006). Social modulation of pain as evidence for empathy in mice. Science, 312, 1967-1970. 
Lefebvre, L. (1982). Food exchange strategies in an infant chimpanzee. Journal of Human Evolution, 11, 195-204.

Lefebvre, L., \& Hewitt, T. A. (1986). Food exchange in captive chimpanzees. In D. M. Taub \& F. A. King (Eds.), Current perspectives in primate social dynamics (pp. ??). Van Nostrand Reihold.

Lonsdorf, E. V., Pusey, A. E., \& Eberly, L. (2004). Sex differences in learning in chimpanzees. Nature, 428, 715-716.

Masserman, J., Wechkin, M. S., and Terris, W. (1964). Altruistic behavior in rhesus monkeys. American Journal of Psychiatry, 121, 584-585.

Matsuzawa, T. (Ed.). (2001). Primate origins of human cognition and behavior. Tokyo: Springer-Verlag Tokyo.

Melis, A. P., Hare, B., and Tomasello, M. (2008). Do chimpanzees reciprocate received favours? Animal Behaviour, 76, 951-962.

Menzel, C. R. (1999). Unprompted recall and reporting of hidden objects by a chimpanzee (Pan troglodytes) after extended delays. Journal of Comparative Psychology, 113, 426-434.

Mitani, J. C. (2006). Reciprocal exchange in chimpanzees and other primates. In P. Kapeller \& C. P. van Schaik (Eds.), Cooperation in primates and humans: Evolution and mechanisms (pp. 101-113). Berlin: Springer.

Mitani, J. C., \& Watts, D. P. (2001). Why do chimpanzees hunt and share meat? Animal Behaviour, 61, 915-924.

Nissen, H. W., \& Crawford, M. P. (1936). A preliminary study of food-sharing behavior in young chimpanzees. Journal of Comparative Psychology, 22, 383-419. 
Ofek, H. (2001). Second nature: Economic origins of human evolution. Cambridge: Cambridge University Press.

Paquette, D. (1992). Object exchange between captive chimpanzees: A case report. Human Evolution, 7, 11-15.

Rumbaugh, D. M. (1977). Language learning by a chimpanzee: The LANA project. New York: Academic Press.

Rumbaugh, D. M., \& Washburn, D. A. (2003). Intelligence of apes and other rational beings. New Haven: Yale University Press.

Savage-Rumbaugh, E. S. (1986). Ape Language: From conditioned response to symbol. New York: Columbia University Press.

Savage-Rumbaugh, E. S., Rumbaugh, D. M., \& Boysen, S. (1978). Linguistically mediated tool use and exchange by chimpanzees (Pan troglodytes). The Behavioral and Brain Sciences, 4, 539-554.

Silk, J. B. (1979). Feeding, foraging and food sharing behavior of immature chimpanzees. Folia primatologica, 31, 123-142.

Silk, J. B., Brosnan, S. F., Vonk, J., Henrich, J., Povinelli, D. J., Richardson, A. S., Lambeth, S., Mascaro, J., and Schapiro, S. (2005). Chimpanzees are indifferent to the welfare of unrelated group members. Nature, 437, 1357-1359.

Slocombe, K. E., \& Newton-Fisher, N. E. (2005). Fruit sharing between wild adult chimpanzees (Pan troglodytes schweinfurthii): A socially significant event? American Journal of Primatology, 65, 385-391.

Smith, A. (2000). The wealth of nations. New York: Random House (Original work published in 1776). 
Sousa, C., \& Matsuzawa, T. (2001). The use of tokens as rewards and tools by chimpanzees (Pan troglodytes). Animal Cognition, 4, 213-221.

Warneken, F., and Tomasello, M. (2006). Altruistic helping in human infants and young chimpanzees. Science, 311, 1301-1303.

Warneken, F., Hare, B., Melis, A. P., Hanus, D., and Tomasello, M. (2007). Spontaneous altruism by chimpanzees and young children. PLoS Biology, 5, e184.

Wechkin, M. S., Masserman, J., and Terris, W. (1964) Shock to a conspecific as an aversive stimulus. Psychonomic Science, 1, 47-48. 


\section{Acknowledgements}

Sarah F. Brosnan (S.F.B), Department of Psychology, Neuroscience Institute, and Language Research Center, Georgia State University; Michael J. Beran (M.J.B.), Language Research Center, Georgia State University. S.F.B. was supported by an NSF Human and Social Dynamics grant (SES 0729244). M.J.B was supported by NICHD grant HD-38051, and NSF grants BCS-0634662 and SES 0729244. The LRC colony was supported by NICHD HD-38051 and the College of Arts and Sciences, Georgia State University. We thank Betty Chan, Theodore Evans, Sarah Hunsburger, and John Kelley for assistance with data collection. Correspondence should be addressed to: Sarah F. Brosnan, Georgia State University, Department of Psychology, PO Box 5010, Atlanta, GA 30302-5010; Email: sbrosnan@gsu.edu 
Table 1: Summary of procedures for each test

\begin{tabular}{|c|c|c|c|}
\hline & $\begin{array}{l}\text { Session \& trial } \\
\text { number }\end{array}$ & Task Description & Goal \\
\hline \multicolumn{4}{|l|}{ Experiment 1} \\
\hline Phase 1 & $\begin{array}{l}7 \text { sessions of } 15 \text { tokens } \\
\quad \text { ( } 5 \text { per food) } \\
5 \text { sessions of } 20 \text { tokens } \\
3 \text { sessions of } 15 \text { tokens }\end{array}$ & $\begin{array}{l}\text { Tokens are given in a bowl and subjects may return tokens in } \\
\text { any order to receive the corresponding food item }\end{array}$ & $\begin{array}{l}\text { Will subjects trade lexigram } \\
\text { tokens for food? }\end{array}$ \\
\hline Phase 2 & $\begin{array}{l}5 \text { sessions with variable } \\
\text { number of foods } \\
\text { available each session }\end{array}$ & $\begin{array}{l}\text { Non-renewable cache of food available with Exp 1. Subject must } \\
\text { choose token from Exp } 2 \text { and exchange with Exp } 1 \text { to obtain } \\
\text { food. Tokens are renewed every trial. }\end{array}$ & $\begin{array}{l}\text { Will subjects limit their return } \\
\text { of tokens to foods which are } \\
\text { available? }\end{array}$ \\
\hline Phase 3 & $\begin{array}{l}15 \text { sessions with } \\
\text { variable number of } \\
\text { foods available each } \\
\text { session }\end{array}$ & $\begin{array}{l}\text { Same as Phase 2, except subjects are tested with a partner and } \\
\text { must discriminate between their cache and their partner's cache. }\end{array}$ & $\begin{array}{l}\text { Will subjects restrict their } \\
\text { barter to foods that are } \\
\text { available for them? }\end{array}$ \\
\hline \multicolumn{4}{|l|}{ Experiment 2} \\
\hline Phase 1 & $\begin{array}{c}8 \text { sessions with variable } \\
\text { number of trials } \\
\text { (session ended when } \\
\text { either subject quit } \\
\text { participating) }\end{array}$ & $\begin{array}{l}\text { Each chimpanzee shown } 1 \text { food and given } 1 \text { token. Half of the } \\
\text { time the token matches the food and the best response is to } \\
\text { exchange. Half of the time the token matches the partner's food } \\
\text { (and the reverse) and the best response is to trade. }\end{array}$ & $\begin{array}{l}\text { Do chimpanzees recognize } \\
\text { when they could maximize } \\
\text { rewards by trading tokens with } \\
\text { a partner? }\end{array}$ \\
\hline Phase 2 & $\begin{array}{l}6 \text { sessions with } 3 \text { pre- } \\
\text { test trials and variable } \\
\text { number of test trials } \\
\text { (session ended when } \\
\text { either subject quit } \\
\text { participating) }\end{array}$ & $\begin{array}{l}\text { Pre-test: each subject was given } 2 \text { tokens and had to return the } \\
\text { one which matched the proffered food to receive it. } \\
\text { Test: each subject was given } 2 \text { tokens, one of which was useless } \\
\text { to both chimps and one of which was useful to either them or } \\
\text { their partner. Subjects had to give one token to their partner, who } \\
\text { had to give one to them, following which they could exchange. }\end{array}$ & $\begin{array}{l}\text { Do chimpanzees succeed more } \\
\text { often when they do not have to } \\
\text { decide whether to trade their } \\
\text { only token, but instead must } \\
\text { decide which of two tokens to } \\
\text { trade? }\end{array}$ \\
\hline
\end{tabular}




\begin{tabular}{|c|c|c|c|}
\hline Phase 3 & $\begin{array}{l}2 \text { sessions with } 3 \text { pre- } \\
\text { test trials and variable } \\
\text { number of test trials } \\
\text { (session ended when } \\
\text { either subject quit } \\
\text { participating) }\end{array}$ & $\begin{array}{l}\text { Same pre-test as Phase } 2 \\
\text { Each chimpanzee is shown a single food. Two tokens are } \\
\text { presented to one of the chimpanzees, one of which matches their } \\
\text { food and one of which matches the partners' food. The subject } \\
\text { must slide a token to their partner and then may exchange the } \\
\text { other. Subjects alternate receiving the tokens. }\end{array}$ & $\begin{array}{l}\text { Do chimpanzees succeed more } \\
\text { often when all of the available } \\
\text { tokens are useful to either them } \\
\text { or their partner? }\end{array}$ \\
\hline Phase 4 & $\begin{array}{l}3 \text { sessions with variable } \\
\text { number of test trials } \\
\text { (session ended when } \\
\text { either subject quit } \\
\text { participating) }\end{array}$ & $\begin{array}{l}\text { Each chimpanzee is shown a single food. Three tokens are } \\
\text { presented to one of the chimpanzees, one which matches their } \\
\text { food, one which matches their partners' food, and one which is a } \\
\text { foil (matches nothing). The subject must slide a token to their } \\
\text { partner and then may exchange the other. Subjects alternate } \\
\text { receiving the tokens. }\end{array}$ & $\begin{array}{l}\text { Will chimpanzees choose a } \\
\text { token required by their partner } \\
\text { when multiple tokens are } \\
\text { available to be traded? }\end{array}$ \\
\hline \multicolumn{4}{|c|}{ Experiment 3} \\
\hline Phase 1 & 2 sessions of 12 trials & $\begin{array}{l}\text { A divided food bonanza is placed in front of the partner. The } \\
\text { subject must choose which token to give to the partner from two } \\
\text { presented on a board. If the token passed to the partner matches } \\
\text { the food bonanza, both individuals receive half of the food. }\end{array}$ & $\begin{array}{l}\text { Will chimpanzees pass tokens } \\
\text { to a partner for their own gain? }\end{array}$ \\
\hline Phase 2 & 4 sessions of 12 trials & $\begin{array}{l}\text { Subjects were next to an empty run, with food in front of them. } \\
\text { The subject received two tokens, and had to push one token to } \\
\text { the empty cage before exchanging the other. This is the same } \\
\text { protocol as Exp 2, Phase 2, except that no partner is present. }\end{array}$ & $\begin{array}{l}\text { Will chimpanzees discard a } \\
\text { useless token before } \\
\text { exchanging the correct token } \\
\text { with an experimenter? }\end{array}$ \\
\hline Phase 3 & 3 sessions of 12 trials & $\begin{array}{l}\text { This is the same protocol as Exp 2, Phase 2, except that one } \\
\text { subject makes all decisions in a given session (rather than } \\
\text { alternating). }\end{array}$ & $\begin{array}{l}\text { Will chimpanzees give a token } \\
\text { which is useless to them to } \\
\text { their partner? }\end{array}$ \\
\hline Phase 4 & 4 sessions of 12 trials & $\begin{array}{c}\text { This is the same protocol as Experiment 2, Phase 1, except in } \\
\text { this case one subject makes all decisions in a given session } \\
\text { (rather than alternating). }\end{array}$ & $\begin{array}{l}\text { Do chimpanzees evaluate } \\
\text { whether token exchange is } \\
\text { appropriate for a given } \\
\text { situation? }\end{array}$ \\
\hline
\end{tabular}




\begin{tabular}{|c|c|c|c|}
\hline Phase 5 & 2 sessions of 12 trials & $\begin{array}{c}\text { For each trial, subjects are given } 4 \text { tokens, } 2 \text { of which can be } \\
\text { exchanged for food and } 2 \text { of which represent foods the partner } \\
\text { has. The experimenter does not enforce trade prior to exchange. }\end{array}$ & $\begin{array}{c}\text { Will chimpanzees barter in an } \\
\text { unrestricted situation? }\end{array}$ \\
\hline
\end{tabular}


Table 2: Do chimpanzees know when to trade with a partner?

For each of the tests involving the opportunity to trade with a partner, we give the absolute number of correct and incorrect trades and the percentage of each (in parenthesis). Here trade indicates the giving of a token to the partner. For descriptions of each test, see Table 1.

\begin{tabular}{|c|c|c|c|c|c|c|}
\hline \multirow[b]{2}{*}{ Experiment } & \multicolumn{2}{|c|}{ Sherman } & \multicolumn{2}{|c|}{ Panzee } & \multicolumn{2}{|c|}{ Lana } \\
\hline & Correctly & Incorrectly & Correctly & Incorrectly & Correctly & Incorrectly \\
\hline 2.2 .1 & $71(76.3 \%)$ & $22(23.7 \%)$ & $65(74.7 \%)$ & $22(25.3 \%)$ & $\mathrm{n} / \mathrm{a}$ & $\mathrm{n} / \mathrm{a}$ \\
\hline 2.2 .2 & $28(100 \%)$ & $0(0 \%)$ & $4(25 \%)$ & $12(75 \%)$ & $8(44.4 \%)$ & $10(55.6 \%)$ \\
\hline 2.2 .3 & $43(97.7 \%)$ & $1(0.3 \%)$ & $1(6.2 \%)$ & $15(93.8 \%)$ & $23(71.9 \%)$ & $9(23.1 \%)$ \\
\hline 2.2 .4 & $6(28.6 \%)$ & $15(71.4 \%)$ & $\mathrm{n} / \mathrm{a}$ & $\mathrm{n} / \mathrm{a}$ & $0(0 \%)$ & $21(100 \%)$ \\
\hline $2.2 .4 \&$ & $13(61.9 \%)$ & $8(38.1 \%)$ & $\mathrm{n} / \mathrm{a}$ & $\mathrm{n} / \mathrm{a}$ & $20(95.2 \%)$ & $1(4.8 \%)$ \\
\hline 3.2 .1 & $23(95.8 \%)$ & $1(4.2 \%)$ & $24(100 \%)$ & $0(0 \%)$ & $21(87.5 \%)$ & $3(12.5 \%)$ \\
\hline $3.2 .2^{*}$ & 42 (87.5\%) & $6(12.5 \%)$ & 41 (85.4\%) & $7(14.6 \%)$ & 37 (77.1\%) & $11(22.9 \%)$ \\
\hline 3.2 .3 & $36(100 \%)$ & $0(0 \%)$ & 29 (80.5\%) & 7 (19.5\%) & 34 (94.4\%) & $2(5.6 \%)$ \\
\hline 3.2.4\# & $46(95.8 \%)$ & $2(4.2 \%)$ & 35 (72.9\%) & $13(27.1 \%)$ & 40 (83.3\%) & $8(16.7 \%)$ \\
\hline 3.2.5@ & $16(88.9 \%)$ & $2(11.1 \%)$ & $\mathrm{n} / \mathrm{a}$ & $\mathrm{n} / \mathrm{a}$ & $12(46.2 \%)$ & $14(53.8 \%)$ \\
\hline
\end{tabular}

\& indicates that 'correctly' involved exchanging both tokens to the partner, rather than just one of them.

* indicates that subject had only to discard the correct token under the mesh, but no partner received it.

\# indicates that the subject had to decide whether or not to trade; correct means that they made the correct decision regardless of whether it involved trade.

@ indicates that, of the trades which were actually made, which were correct and which were incorrect. Note that to get the maximum amount of food, if they understood trade, each subject should have traded 2 tokens on every trial for a total of 48 trades. Neither subject is anywhere close to this maximum number, indicating that in most cases, they chose not to trade (not indicated on table). 
